\title{
Postgraduate Education in the Netherlands
}

In the spring of this year the Dutch Association of Logopedics organized a scientific meeting of which lasted 2 days, and was meant to prepare the International Congress of the IALP 1974 at Interlaken, by focussing on topics of the main reports.

The subject of the meeting was 'The Prevention of Developmental Speech and Language

Disorders'.

Developmental Language Disorders as a Cause of Learning-Disabilities

J. J. Dumont, University of Nijmegen, Nijmegen

Summary

Besides demonstrable neurological defects, defective sensorimotor or visuospatial development are often responsible for dyslexia and dysorthography.

In more than $50 \%$ of the cases, auditory aspects of language development were found to be involved. Analysis and diagnosis of the symptoms may lead to assessment of these factors and to an adequate individual treatment program.

The Influence of Minimal Brain Damage on the Development and Language Development of Young Children

A. F. Kalverboer, University of Groningen, Groningen

Summary

The severity of the brain damage may be in no proportion to the disabilities, which depend mainly on the age at which the damage took place. Before the age of 2 years, there will be no damage to the function of language development. After 4 years of age, these functions are defined to typical parts of the brain (Lenneberg).

Dysfunctions caused by brain damage are: (1) integration of sensory input (rhythmic and simultaneous); (2) inhibition of the selecting system (motor function, concentration), and (3) channel capacity (Eisenson).

We may therefore conclude that there is a direct influence of brain damage in retardation of function development, and an indirect influence of brain damage on selective attention for sounds and inconsistent behaviour. Observation in crucial situations and testing the capacity for sound differentiation can inform us about the relation between total behaviour and language behaviour. This is a basis for diagnosis.

Societates

399

Early Discovery of Speech-Impaired Children

J. van Dijk, Institute for the Deaf, Vught

Summary

This paper centered around the following points of discussion: (1) language seen as a linguistic system or as a means for exchange of thought; (2) the role of the parents in the process of language acquisition, as found by Brown and Bellugi (imitation, expansion and correction); (3) is learning to speak a process of operant conditioning? (4) how might the relation be between thought and speech? Is language a condition for thinking? 
Other plans for postgraduate education in the Netherlands: (1) attending lessons and special treatment sessions at the training centers for speech therapists; (2) attending study meetings and lectures organized by divisional committees of the Dutch Society for Logo-pedics; (3) doing terms in hospitals and institutions or with individual speech therapists; (4) a central library is at disposal which mails out professional literature.

There was a discussion about how to meet the need for advanced special training in the University and for preparing the speech therapist for a university degree, as is already realized in some other countries, e.g. in Belgium.

An important condition for further development and reasearch in speech pathology is multidisciplinary training of university graduates in the medical and psychological sciences. Also the phoniatric field must be developed. Scientific endeavour in narrow cooperation with the speech therapist will be essential, as described by Arnold and Luchsinger and by Biesalski. In the center of this combined field, our patients are waiting to profit from our joint future achievements. We also need more intensive international contact, writing and speaking the same professional language. 